\title{
Effect of Bileaflet Valve Orientation on the 3D Flow Dynamics in the Sinus of Valsalva*
}

\author{
Takanobu YAGI**, William YANG*** and Mitsuo UMEZU** \\ ${ }^{* *}$ Center for Advanced Biomedical Sciences, TWIns, Waseda University \\ 03C204 2-2 Wakamatsucho Shinjukuku, Tokyo 162-8480, Japan \\ ${ }^{* * \star}$ CSIRO Process Science and Engineering, Box312, Clayton South, Victoria 3169, Australia \\ E-mail: takanobu_yagi@aoni.waseda.jp
}

\begin{abstract}
Three-dimensional (3D) evolutional behavior of flow dynamics in the aortic sinus of Valsalva was studied in vitro for finding the effects of bileaflet valve orientations in an effort to investigate possible linkages between the sinus flow and coronary circulation. A realistic model of the aortic sinus was machined inside an acrylic block. A St. Jude Medical bileaflet aortic valve was utilized. Two orientations were compared; (1) a pair of hinges aligned in the direction of the non-coronary sinus of the model (N0) and (2) of the left and right sinuses (N90). A 3D scanning three-component velocity measurement, named Fluorescent Scanning Stereoscopic Particle Image Velocimetry (FS-SPIV), was developed. Measured data were validated with an aid of two-dimensional particle image velocimetry. Good agreement confirmed the capability of quantifying the 3D mean and turbulent behavior of sinus vortices. The valve orientation was found to be unable to affect the sinus vortices during peak-systole, but had a substantial impact on regurgitation during end-systole. The superior case (N0) gave a symmetric regurgitation. The regurgitant jet was directed to the non-coronary sinus. The left and right sinuses were dominated by a well-organized recirculation from a persistent forward flow. The inferior case (N90) produced an asymmetric regurgitation with a biased jet into the left or right sinuses, leading to produce unexpected turbulence near the entrance of coronary artery. Those results suggest possible linkages between the sinus flow and coronary circulation, thereby advocating the existence of an optimal orientation of bileaflet valves.
\end{abstract}

Key words: Stereoscopic Particle Image Velocimetry, Turbulence, Validation, Aorta, Valsalva, Bileaflet, Orientation, Coronary Circulation

\section{Introduction}

The fluid mechanics of the sinus of Valsalva is known to play a critical role not only for elucidating ideal procedures in aortic root surgery, but also pursuing the optimal design and usage of prosthetic heart valves. An aortic valve consists of three leaflets, which are connected with their own sinuses at the rear side. In 1969, Bellhouse and Talbot revealed that the aortic sinus promotes the formation of a vortex flow behind the valve leaflet, thereby having a functional relationship with valve opening and closing dynamics. ${ }^{(1)}$ The vortex flow during a decelerating phase is found to facilitate the valve closure, resulting in minimizing a transvalvular regurgitation. Since their pioneering work, however, the role of aortic sinus has been failed to be fully understood yet, particularly on possible linkages with the coronary circulation. ${ }^{(2-5)}$

*Received 24 Jan., 2011 (No. 11-0067) [DOI: 10.1299/jbse.6.64]

Copyright $@ 2011$ by JSME 
Magnetic resonance velocity mapping is capable of visualizing sinus flows in vivo. ${ }^{(6-7)}$ Normal subjects can give the organized formation of sinus vortices toward the end of systole, whereas patients treated with aortic root surgery fail to show such organized evolutions. In fact, the sinus flows become more disorganized toward end-systole. ${ }^{(8)}$ Although interpreting the clinical significance of those man-made flows may require further investigations, those data allow us to examine further the value of the fluid mechanics of aortic sinus.

The present research was designed by referring Kleine et al., who investigated the effect of prosthetic aortic valve types and orientations on coronary circulation in vivo by animal experiments, leading to demonstrate the existence of an optimal orientation. ${ }^{(9)}$ In the case of a bileaflet valve, the coronary flow rate is found to increase if a pair of hinges is aligned in the direction of the non-coronary sinus. The flow rate varies by nearly $40 \%$ between the superior and inferior orientations. Downstream turbulences and transvalvular pressure gradients of both the superior and inferior orientations were measured in vivo, resulting in a clear difference between them. ${ }^{(10-11)}$

For better understanding the relationship between the sinus flow and coronary circulation, this study aimed to investigate that the time-dependent evolution of sinus flows from mid-systole to end-systole could be affected by altering valve orientations. The well-organized evolution of the sinus vortices was believed to play a key role for promoting the coronary circulation, because the onset of the mainstream coincides with the closure of aortic valve. This study developed a state-of-the-art 3D scanning three-component velocity measurement technique, named Fluorescent Scanning Stereoscopic Particle Image Velocimetry (FS-SPIV). Replacing a tricuspid valve with a bileaflet valve requires an appropriate alignment to three sinuses, leading to increase the three-dimensional asymmetric nature of aortic flow. On the top of the complexity, turbulence appears and varies regionally as interacted with valve opening and closing dynamics. A full understanding of the spatial and temporal evolution of such complex flows is beyond the ability of conventional point-to-point or planar measurements, such as a hot-film anemometer, laser doppler velocimetry, and particle image velocimetry (PIV). ${ }^{(12-15)}$ MRI 3D velocity mapping show the possibility of quantifying fluctuating velocities in vivo. ${ }^{(16)}$ Future work should include comparisons of the obtained data from the mapping procedure with those of in vitro actual measurements in defined conditions, because fluctuating velocities, unlike mean components, tend to be readily biased by the lack of spatial and temporal resolution as well as dynamic range. The present study, for the first time, quantitatively demonstrates the three-dimensional time evolution of sinus vortices for both mean and turbulent behaviors during a decelerating phase for better understanding the fluid mechanics of the sinus of Valsalva, especially in terms of the relationship with coronary circulation.

\section{Methods}

\subsection{A model of the aortic sinus}

Figure 1 shows a model of the aortic sinus, which was machined inside an acrylic block. The sinus geometry as shown in Fig. 2 was determined by referring to human data. ${ }^{(17-18)}$ The straight conduit was placed at the upstream and downstream of the sinus. This idealized condition ensured that the inlet flow was axisymmetric and the outlet flow was unaffected by the downstream curvature. Inherent complexities observed in vivo, such as the effect of flow eccentricity at the aortic root, the existence of aortic arch, and the effect of wall compliance, were excluded. A St. Jude Medical bileaflet aortic valve was installed, and two types of orientations (N0 and N90) were compared as performed by Kleine et al. ${ }^{(9)}$ In view of the small fraction of coronary flow rate (approximately $60 \mathrm{~mL} / \mathrm{min}$ in the left coronary 
artery at rest ${ }^{(19-20)}$ ) to the total cardiac flow, the existence of coronary flow was assumed to be unable to affect the time-dependent evolution of sinus flows. This assumption was reinforced by the fact that the major coronary flow begins immediately after valve closure. The sinus flow pattern was believed to be dominated by the regurgitation of the mainstream due to the adverse pressure gradient. The geometries of three sinuses were assumed to be identical, which were assigned to the non-coronary $(\mathrm{N})$, left $(\mathrm{L})$, and right $(\mathrm{R})$ sinuses in accordance with the valve orientation (Fig. 2).

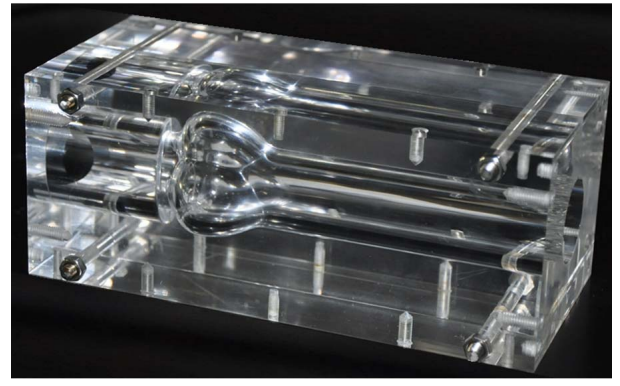

Fig. 1 An acrylic model of aortic sinus

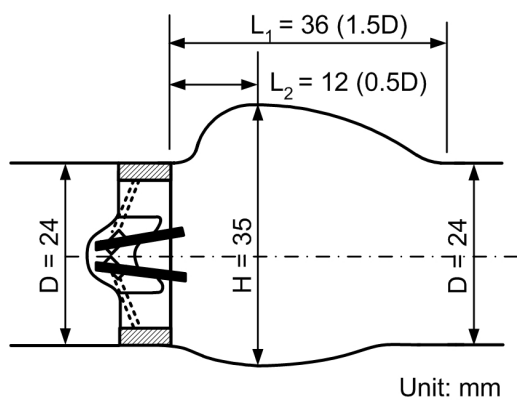

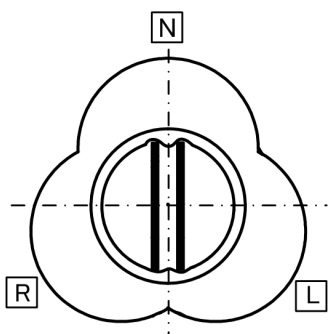

No

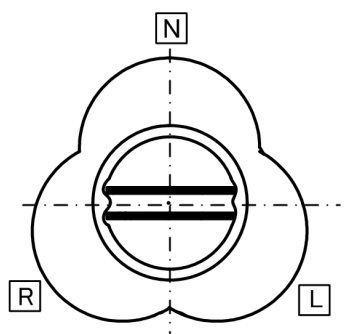

N90

Fig. 2 Geometry of the aortic sinus model (left: side view). Two types of valve orientations (N0 and N90) were compared (right: back view). Zero and ninety indicate the angle of hinge in the direction of the non-coronary sinus.

\subsection{Pulsatile flow system}

Figure 3 illustrates a pulsatile flow system with a FS-SPIV system. An open-loop Windkessel circuit was adopted using a model of left ventricle, which was a pneumatically-driven membrane pump. At a pump inlet, a one-way valve was installed, and at the downstream, an aortic sinus was placed. The aortic compliance was imitated by a flexible home-made silicone tube (I.D.: $20 \mathrm{~mm}$, O.D.: $22 \mathrm{~mm}$, length: $250 \mathrm{~mm}$ ). Aortic flow rate and pressure curves were shown in Fig. 4. The flow setting was as follows; a heart rate of 75 beats $/ \mathrm{min}$, a mean flow rate of $5.0 \mathrm{~L} / \mathrm{min}$, and a systolic fraction of $35 \%$. The Reynolds and Womersley numbers were 4000/1100 (peak/mean) and 18.5, respectively.

The working fluid consisted of saturated aqueous sodium iodide, glycerol, and distilled water with a volume fraction of 79:20:1. This fluid is known to give a Newtonian property, and the density and kinematic viscosity were $1700 \mathrm{~kg} / \mathrm{m}^{3}$ and $3.3 \times 10^{-6} \mathrm{~m}^{2} / \mathrm{s}$, respectively. The kinematic viscosity corresponded to that of the blood at the shear rate more than 200 $1 / \mathrm{s}^{(21)}$ The refractive index of acrylic material was found to be $n=1.494$ at a wavelength of $532 \mathrm{~nm}$ at room temperature. The index of the working fluid was precisely maintained to be $n=1.494 \pm 0.002$ at the same conditions during experiments using a multiple wavelength Abbe refractometer (DR-M2)(ATAGO, Tokyo, Japan). 


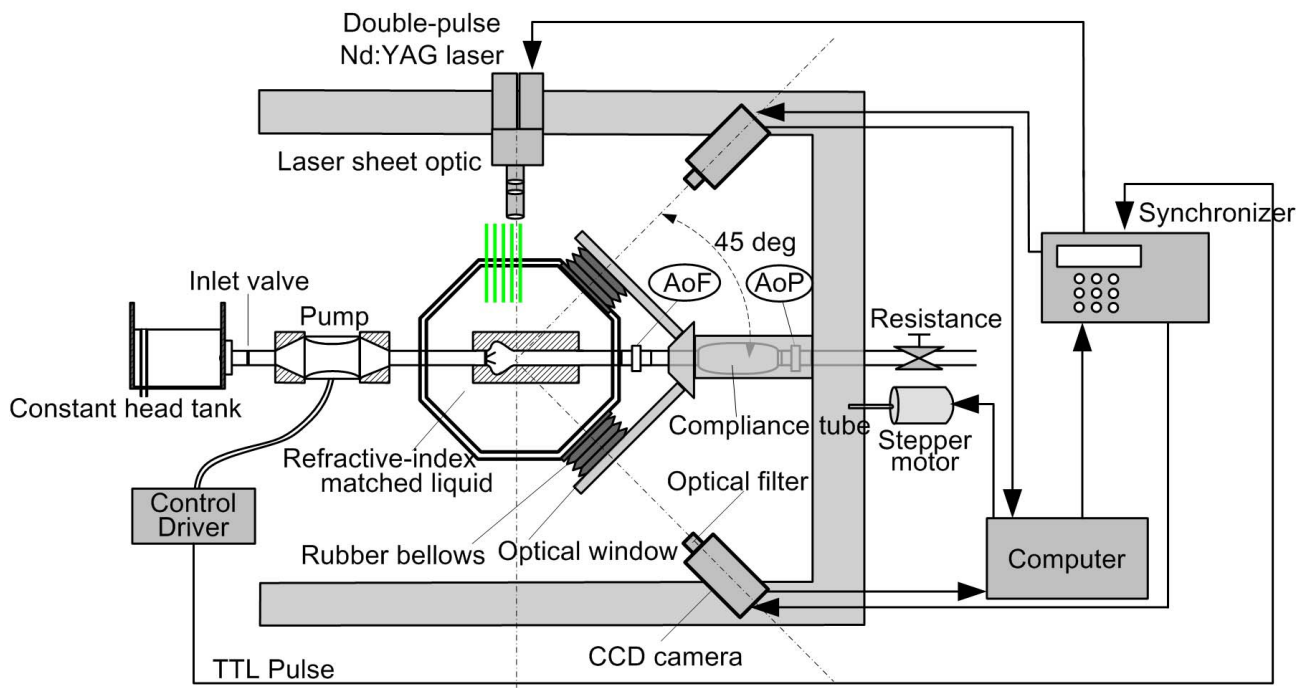

Fig. 3 Schema of a pulsatile flow circuit and a FS-SPIV system
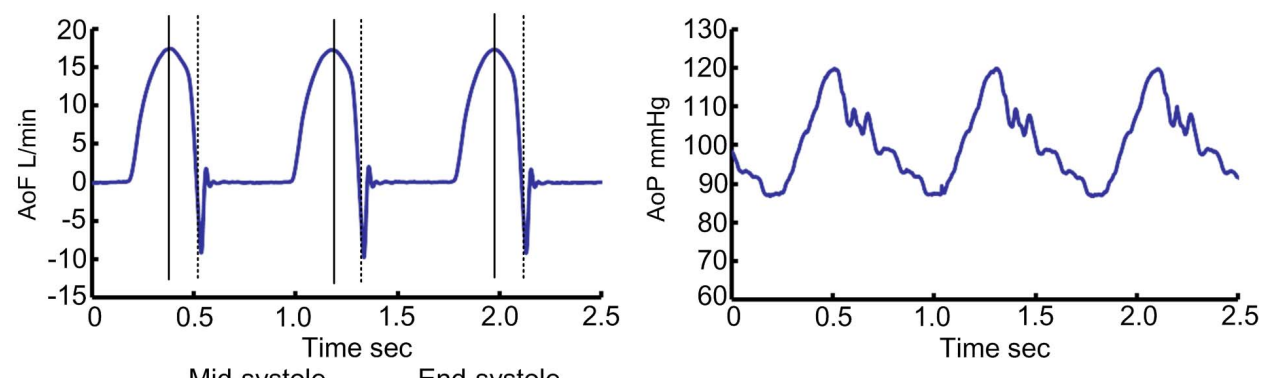

Fig. 4 Aortic flow and pressure waveforms

\subsection{FS-SPIV}

FS-SPIV is a 3D scanning technique of Stereo PIV that can measure a three-component planar velocity field. PIV measures a two-component planar velocity, while SPIV can measure the third (out-of-plane) component, thus being more suited to gain the three-dimensional understanding of complex flows. However, SPIV requires a precise in-situ calibration whose accuracy is directly linked with measurement errors. Since cardiovascular flows are enclosed by complex geometries, the calibration of placing a target plate into the measurement plane is limited or often unfeasible. Some researchers have utilized SPIV for biomedical applications, ${ }^{(22-24)}$ but the efficacy of measuring the third-component velocity is only shown in limited planes. To date, the reconstruction of the 3D volume structures of complex flows has failed to be accomplished yet. FS-SPIV was developed to allow us to perform 3D scanning measurements of SPIV within a complex geometry without repeating a tedious in-situ calibration. Multiplane three-component velocity data sets can reconstruct 3D volume flow structures. A FS-SPIV system consisted of a pair of CCD (charge coupled device) cameras $(1024 \times 1280$ pixels, SensiCam QE Double Shutter)(PCO, Kelheim, Germany) and a double-pulsed Nd:YAG laser (120 $\mathrm{mJ} /$ pulse, Solo PIV)(New Wave Research, Fremont, CA, U.S.A.), and the both were mounted on a U-shaped translational stage equipped a stepper motor (Fig. 3).

For keeping optical parameters constant throughout any scanning plane, an optical system named dynamic prism was developed (Fig. 5). Plane $N$ and $N+1$ correspond to the scanning plane. A sinus model was placed in an octagonal tank, which was equipped with a movable window via a flexible bellows. The optic alignment was connected with the 
translational stage (Fig. 3). Thus, the laser sheet, optical windows, and cameras were linked together, and their relative position was secured throughout any scanning plane. The tank was filled with the index-matched liquid as described above. Then, the following formula was able to be preserved,

$$
\begin{gathered}
\frac{\Delta L_{n 1, N}}{\Delta L_{n 2, N}}=\frac{\Delta L_{n 1, N+1}}{\Delta L_{n 2, N+1}} \\
\Delta d_{L L S}=\Delta d_{w i n}=\Delta d_{l e n s}=\Delta d_{C C D} \\
\theta_{N}=\theta_{N+1}
\end{gathered}
$$

where $\Delta L_{n 1, N}$ and $\Delta L_{n 2, N}$ denote the optical lengths between the laser sheet plane and window plane, and the window plane and sensor plane, respectively, in the $N^{\text {th }}$ scanning plane along with a tilted viewing angle (a lens plane tilted by an angle $\theta_{N}$ to the laser sheet plane as shown in Fig. 5). The subscript of $n 1$ and $n 2$ represent the refractive index of the aforementioned liquid and air, respectively. $\Delta d_{L L S}, \Delta d_{\text {win }}, \Delta d_{\text {lens }}$, and $\Delta d_{C C D}$ correspond to a displacement of each optical element in the direction of scanning. Fulfilling a set of above formula ensures that optical parameters were kept constant throughout any scanning plane. For ensuring particle images in focus over entire field of views from angled viewing, the sensor plane was slightly tilted to the lens plane (Scheimpflug alignment).

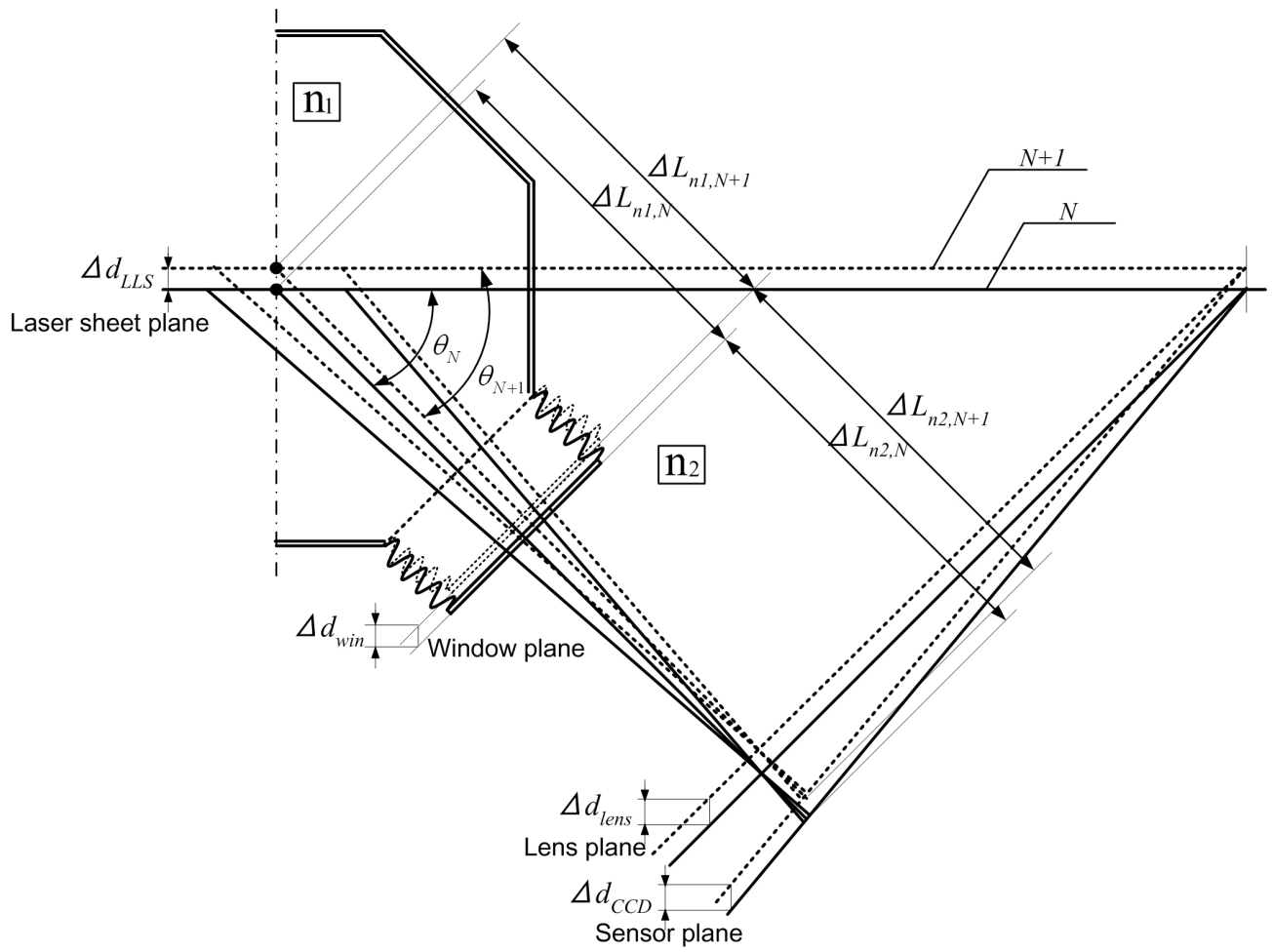

Fig. 5 Schema of dynamic prism

The reconstruction of three-component velocity could be made by any algorithm available. In the present study, the following relationship was solved for $(u, v, w)$ using a least square method, ${ }^{(25)}$ 


$$
\left[\begin{array}{l}
U^{L} \\
V^{L} \\
U^{R} \\
V^{R}
\end{array}\right]=\left[\begin{array}{ccc}
\frac{\partial F_{X}^{L}}{\partial x} & \frac{\partial F_{X}^{L}}{\partial y} & \frac{\partial F_{X}^{L}}{\partial z} \\
\frac{\partial F_{Y}^{L}}{\partial x} & \frac{\partial F_{Y}^{L}}{\partial y} & \frac{\partial F_{Y}^{L}}{\partial z} \\
\frac{\partial F_{X}^{R}}{\partial x} & \frac{\partial F_{X}^{R}}{\partial y} & \frac{\partial F_{X}^{R}}{\partial z} \\
\frac{\partial F_{Y}^{R}}{\partial x} & \frac{\partial F_{Y}^{R}}{\partial y} & \frac{\partial F_{Y}^{R}}{\partial z}
\end{array}\right]\left[\begin{array}{l}
u \\
v \\
w
\end{array}\right]
$$

where $U^{L}, V^{L}, U^{R}$, and $V^{R}$ denote image velocities, and the superscripts, $L$ and $R$, indicate the data that are taken from the left and right cameras, respectively. $X$ and $Y$, and $x, y$, and $z$ correspond to the image and physical coordinate, respectively. The mapping function $F$ was obtained from calibration data. The calibration was made in situ by immersing a target plate into the index-matched liquid. Matching of refractive index allowed us to perform the calibration outside the aortic sinus model. Calibration images were taken at 5 levels within a 1-mm thick laser light sheet. The target plate was positioned by a manual linear stage with a precision micrometer. Then, the mapping function was fitted using the third and second-order polynomials in the in-plane and out-of-plane direction, respectively.

Seeding particles were rhodamine B fluorescent particles (FLUOSTAR)(EBM, Tokyo, Japan). The mean diameter and density were $18 \mu \mathrm{m}$ and $1500 \mathrm{~kg} / \mathrm{m}^{3}$, respectively. A pair of cameras was equipped with a long-pass filter to cut off the reflective lights and selectively pass the fluorescence in order to enhance the signal-to-noise ratio in near-wall regions. The interrogation area was sequentially reduced to $32 \times 32$ pixels $\left(1.9 \times 1.9 \mathrm{~mm}^{2}\right)$ with $75 \%$ overlapping. The processing described above was performed by commercial software (VidPIV 4.6)(Intelligent Laser Application, Jülich, Germany). Measurements were repeated in a phase-locked manner through 400 pulsatile cycles during mid and end-systole. The mean and turbulent kinetic energy were obtained by the following formula,

$$
\begin{gathered}
\bar{u}=\frac{1}{N} \sum u \\
u^{\prime}=\left(\frac{1}{N} \sum(u-\bar{u})^{2}\right)^{0.5} \\
k=\frac{1}{2}\left(u^{\prime 2}+v^{\prime 2}+w^{\prime 2}\right)
\end{gathered}
$$

where $u, v$, and $w$, and $k$ correspond to the three components of the instantaneous velocity vector and turbulent kinetic energy. The bar and prime denote the ensemble-averaged and fluctuating components, respectively. The number of velocity sampling $(\mathrm{N}=400)$ was confirmed to be sufficient to converge the statistics of each quantity.

\section{Results}

\subsection{Measurement validation}

2D-PIV was performed in the same pulsatile flow conditions using the same optical 
hardware. A pair of valve hinges was aligned in the direction of the left and right sinuses (N90). Figure 6 shows the schema of optical alignments of FS-SPIV and 2D-PIV. The axial and radial mean component $(\bar{w}, \bar{v})$ and the fluctuating axial velocity $\left(w^{\prime}\right)$ as measured by FS-SPIV were compared with those by 2D-PIV at various locations along with the $z$ axis. The time phase corresponded to mid-systole $(t / T=0.31)$ where $t$ and $T$ denote the time elapsed from the onset of systole and the cycle period, respectively. The interrogation size of 2D-PIV was sequentially reduced to be $16 \times 16$ pixels $\left(0.7 \times 0.7 \mathrm{~mm}^{2}\right)$ with $50 \%$ overlapping. The thickness of the laser light sheet was approximately $1 \mathrm{~mm}$, similar to that of FS-SPIV.

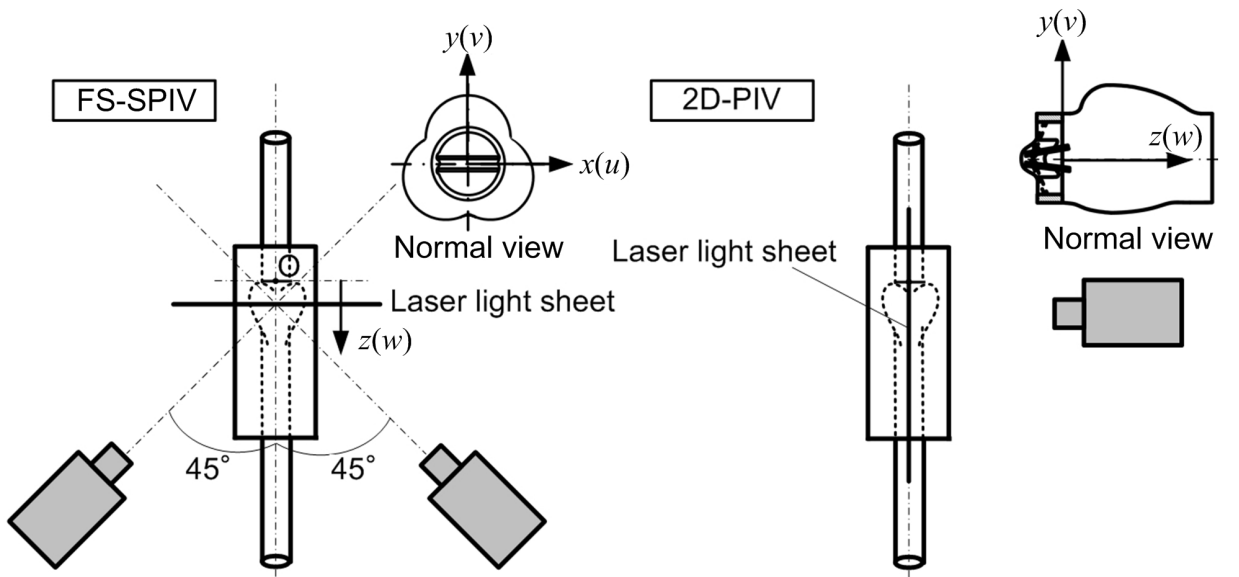

Fig. 6 Schema of FS-SPIV and 2D-PIV alignments
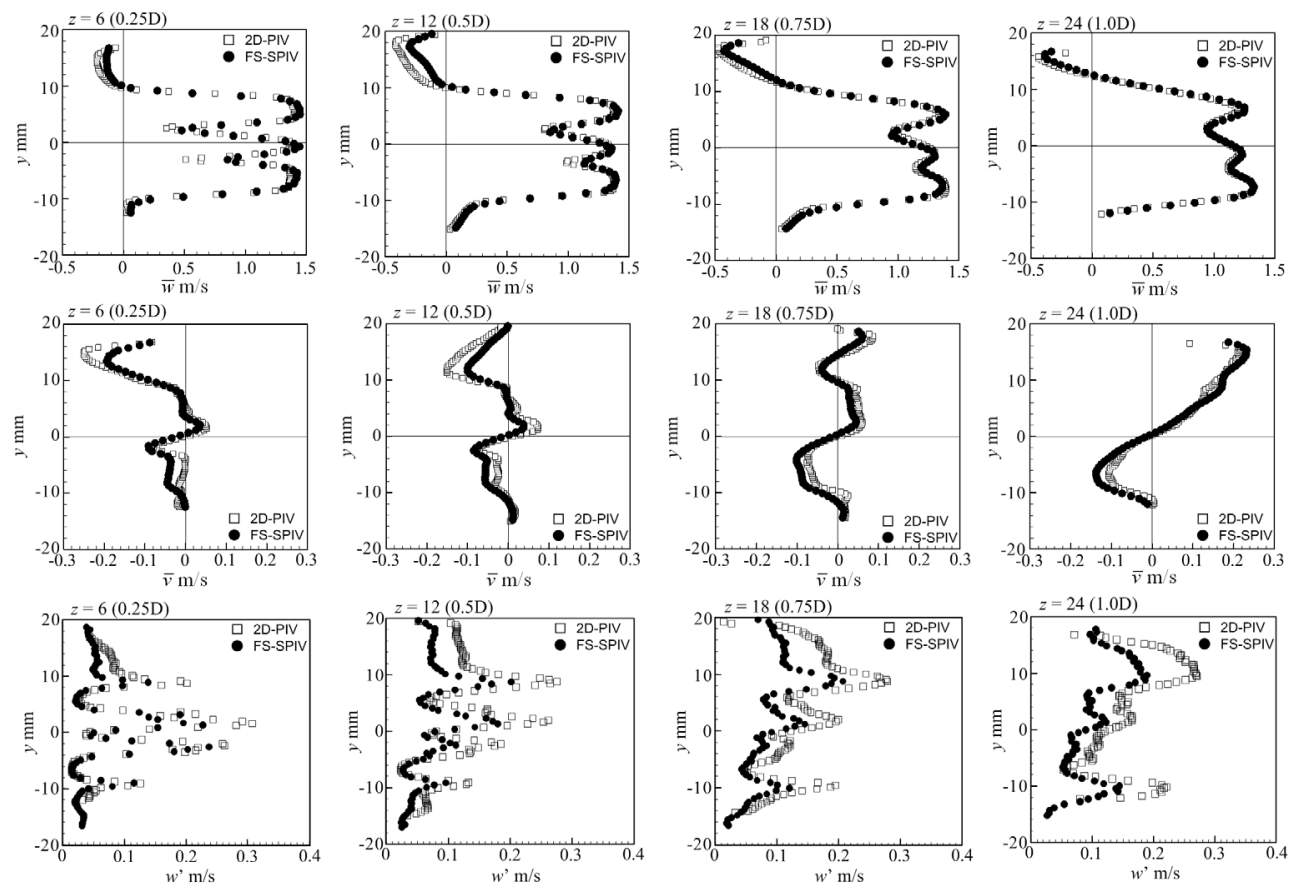

Fig. 7 Comparison of FS-SPIV and 2D-PIV in the axial and radial mean velocity $(\bar{w}, \bar{v})$ and the fluctuating axial velocity $\left(w^{\prime}\right)$ at several locations along with the $z$ axis $(x=0 \mathrm{~mm})$ 

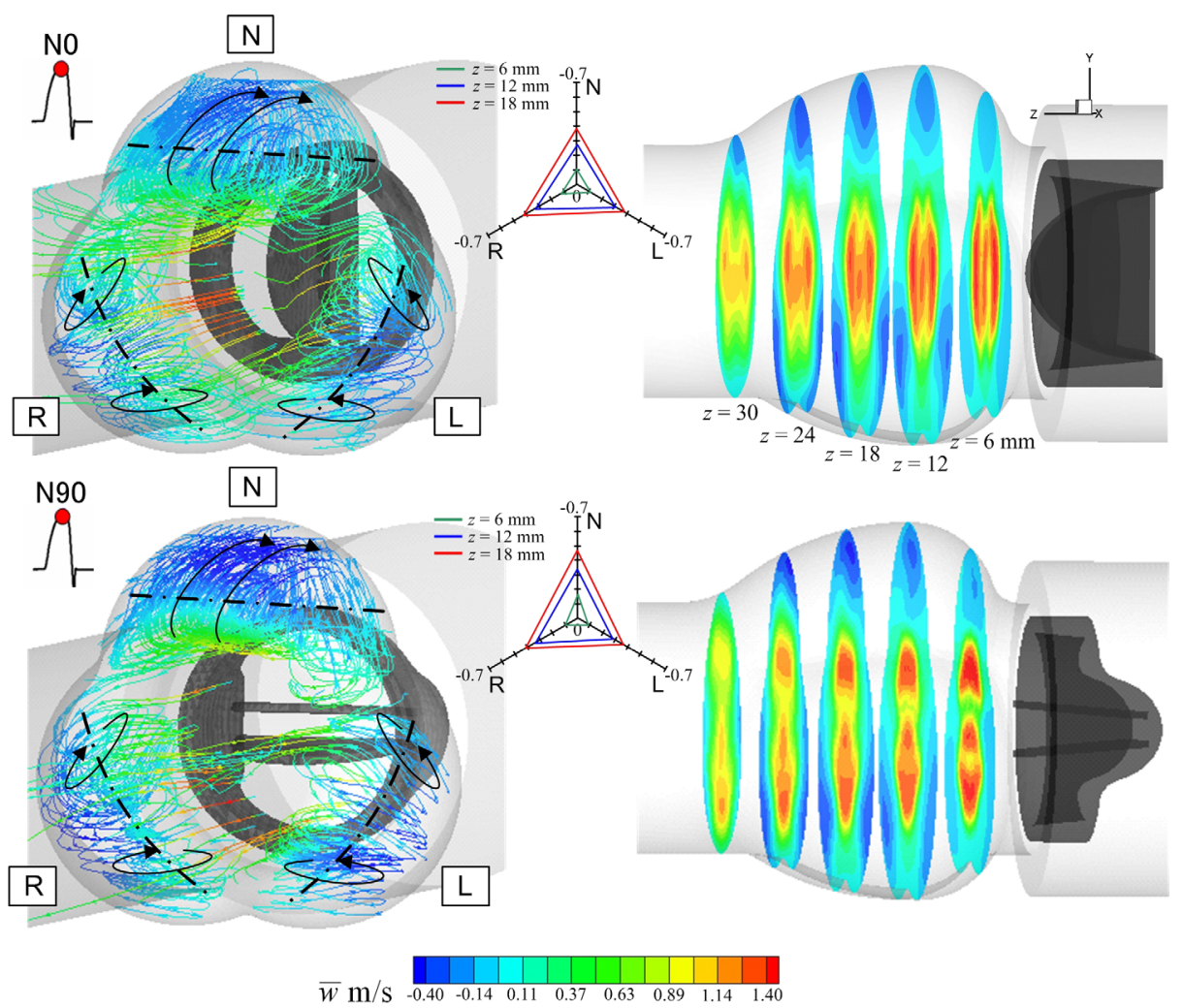

Fig. 8 Comparison of valve orientations (N0 and N90) in 3D streamlines during mid-systole (left) and the corresponding axial velocity profiles at several planes (right). The ternary diagram in middle demonstrates the maximum backflow velocity at $z=6,12$, and $18 \mathrm{~mm}$.

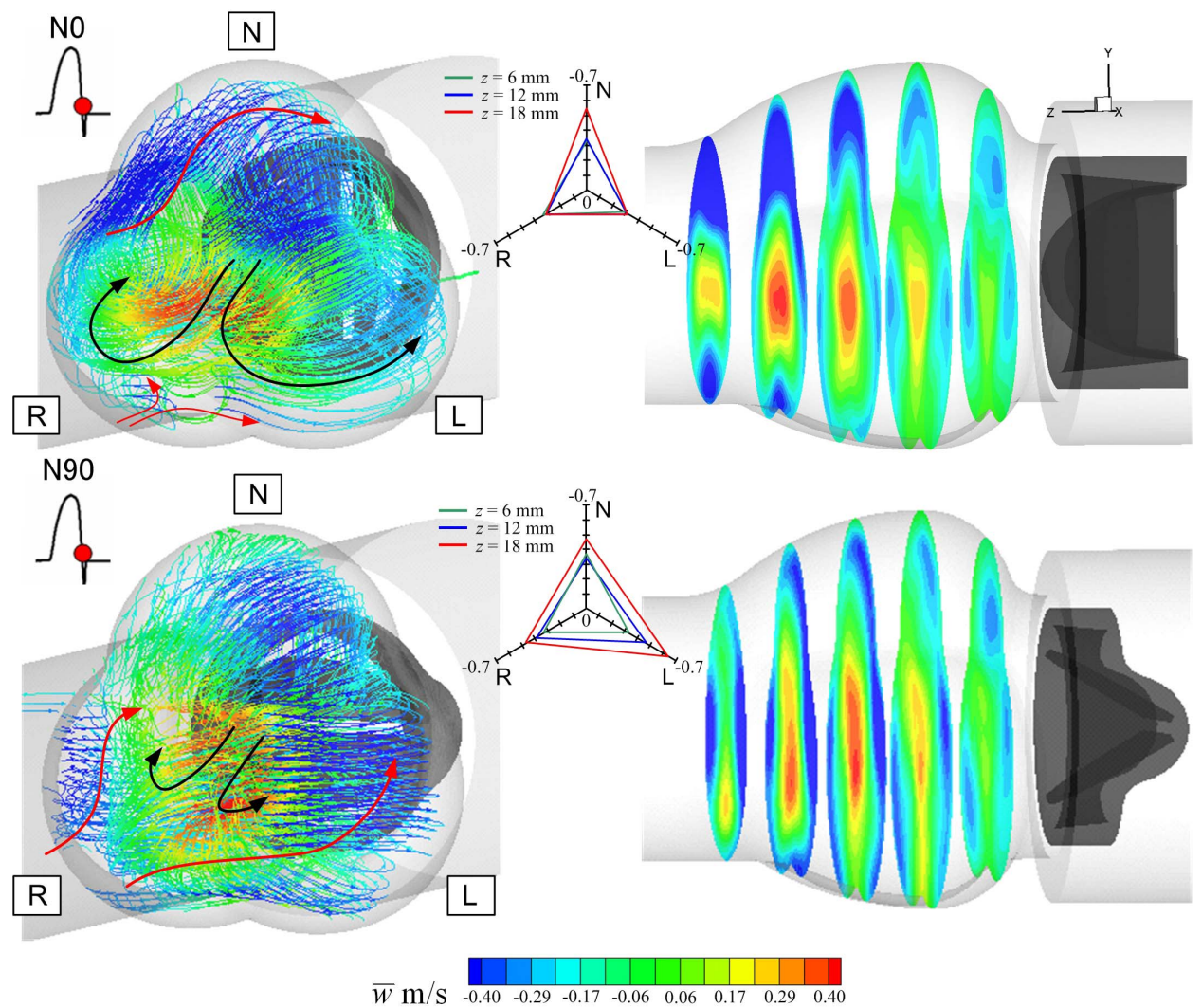

Fig. 9 Comparison of valve orientations (N0 and N90) in 3D streamlines during end-systole (left) and the corresponding axial velocity profiles at several planes (right). The ternary diagram in middle demonstrates the maximum backflow velocity at $z=6,12$, and $18 \mathrm{~mm}$. The solid red and black lines correspond to the regurgitant jet and the persistent forward flow, respectively. 
Mean velocities measured by FS-SPIV and 2D-PIV were found to agree very well (Fig. 7). The axial velocity by FS-SPIV corresponded to the third component reconstructed from in-plane velocities. Good agreement was also confirmed in the out-of-plane velocity. The systematic bias along with the $z$ axis was invisible, confirming that the optical parameter remained constant throughout scanning planes. The fluctuating axial velocity of FS-SPIV reasonably agreed with that of 2D-PIV. FS-SPIV exhibited a similar trend to that of 2D-PIV along with the $y$ axis. Quantitatively, however, some discrepancies were confirmed to exist between them. The largest difference of fluctuating velocity reached several tens of percents. One of the plausible reasons is due to an inherent error source of 2D-PIV, named a perspective projection. ${ }^{(26)}$ The two-component velocity measured by $2 \mathrm{D}-\mathrm{PIV}$ is known to be biased by three-dimensional fluid motions. Three-dimensionality of the flow increased further in the fluctuating velocity than in the mean velocity, because the velocity fluctuation derived from the appearance of turbulent eddies. Yoon and Lee compared 2D-PIV and SPIV in the flow measurement behind an axial fan, and reported that the turbulence intensity by 2D-PIV is overestimated in comparison with that of SPIV due to the perspective error. ${ }^{(27)}$ Thus, the perspective error of 2D-PIV was believed to overestimate the fluctuating velocity.

Other than the perspective error, there may be several factors associated with the discrepancy between FS-SPIV and 2D-PIV. The spatial resolution of FS-SPIV was larger than that of 2D-PIV (FS-SPIV: $1.9 \times 1.9 \mathrm{~mm}^{2}$, 2D-PIV: $0.7 \times 0.7 \mathrm{~mm}^{2}$ ). The larger resolution may smooth the measured velocity. The lack of spatial resolution becomes more remarkable in the fluctuating velocity than in the mean velocity. For fully resolving turbulent eddies, the size of interrogation volume has to be small enough, otherwise the measured velocity becomes spatially averaged. Also, the lack of dynamic range was suspected. The measurement uncertainty in determining the particle displacement by cross correlation is of the order of 0.1 pixels. ${ }^{(2)}$ The image magnification of FS-SPIV was approximately $58.8 \mu \mathrm{m} /$ pixel. The time separation between two laser pulses was set to be $130 \mu \mathrm{s}$. Thus, the particle displacement with a convective velocity of $1 \mathrm{~m} / \mathrm{s}$ corresponded to 2.2 pixels within the time separation. The measurement uncertainty reached $4.5 \%$ approximately, which was found to be nearly twice larger than that of 2D-PIV. Therefore, the measurement uncertainty of fluctuating velocity by FS-SPIV can be estimated to reach several tens of percents. The error analysis above excluded the possible benefit of fluorescent particles that could enhance the signal-to-noise ratio. The future work would address whether fluorescent particles can improve the measurement uncertainty of cross correlation. If the further confidence for the accuracy of turbulent measurements is required, the spatial resolution and dynamic range of FS-SPIV should be improved. Consequently, the results presented here, together with the aforementioned error analyses, demonstrated that the measurement accuracy of FS-SPIV was validated for both mean and turbulent quantities in actual environments.

\subsection{Mean profiles during mid-systole}

Figure 8 shows the comparison of sinus flows between two valve orientations (N0 and N90) as measured by FS-SPIV during mid-systole $(t / T=0.31)$. Measurements covered 12 scanning planes with an increment of $3 \mathrm{~mm}(z=3$ to $36 \mathrm{~mm})$. Three-component velocity data sets over multiple planes were interpolated into $3 \mathrm{D}$ numerical meshes with adequate resolution. Resulting volume data enabled the 3D phase-locked analyses of complex flows. For clear visualization, the streamlines of mainstream were omitted. Interestingly, there was no marked difference between N0 and N90. The sinus vortices were formed in each of three sinuses independently. Each of the sinuses was occupied by a single vortex, and vortices in the left and right sinuses were twisted in accordance with the curvature. The ternary diagram of maximum backflow velocity in each of three sinuses were plotted along with the 
$z$ axis $(z=6,12$, and $18 \mathrm{~mm})$. Those demonstrated that the sinus vortices were axisymmetric along with the $z$ axis.

\subsection{Mean profiles during end-systole}

Figure 9 shows the comparison of sinus flows (N0 and N90) during end-systole $(t / T=$ 0.49 ). The onset of the coronary flow occurs immediately after this time phase. Thus, the corresponding sinus flow was believed to be related with the coronary circulation. Unlike mid-systole, the sinus flows of N0 during end-systole were found to differ significantly from that of N90. In the case of N0, the regurgitant jet was directed to the non-coronary sinus. The left and right sinuses were dominated by a well-organized recirculation from a persistent forward flow. In the case of N90, the regurgitant jet was directed to the N-L and $\mathrm{N}-\mathrm{R}$ sinus commissures rather than the non-coronary sinus. The regurgitant jet squeezed a persistent forward flow. The velocity profile of the forward flow was stretched along with the $y$ axis (Fig. 9), though that of N0 remained to be circular. The stretched flow patterns of N90 resulted in asymmetric distributions of the sinus flow. The regurgitant jet was biased to the left coronary sinus. This skewed flow pattern was thought to be able to readily invert by the slight alteration of valve orientation. In other words, the regurgitant flow pattern of N90 was quite unstable as affected by positioning the valve orientation at the aortic root. The ternary diagram of maximum backflow velocity demonstrated that $\mathrm{N} 0$ orientation allowed the symmetric regurgitation to appear in the left and right sinus, whereas N90 resulted in the asymmetric skewed regurgitation. The asymmetric nature of velocity magnitude became more remarkable as it closed to the sinotubular junction.

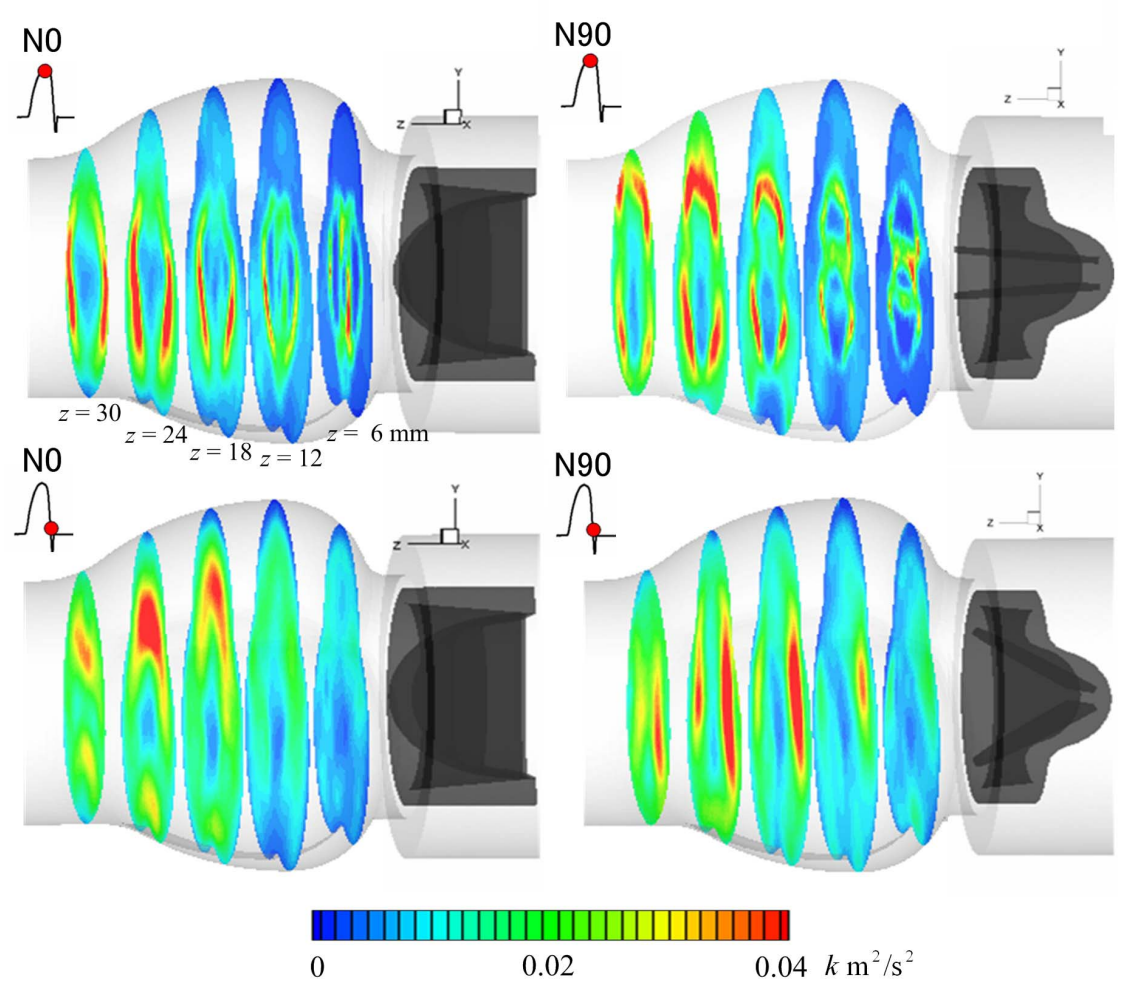

Fig. 10 Comparison of valve orientations (N0 and N90) in turbulent kinetic energy during mid and end-systole 

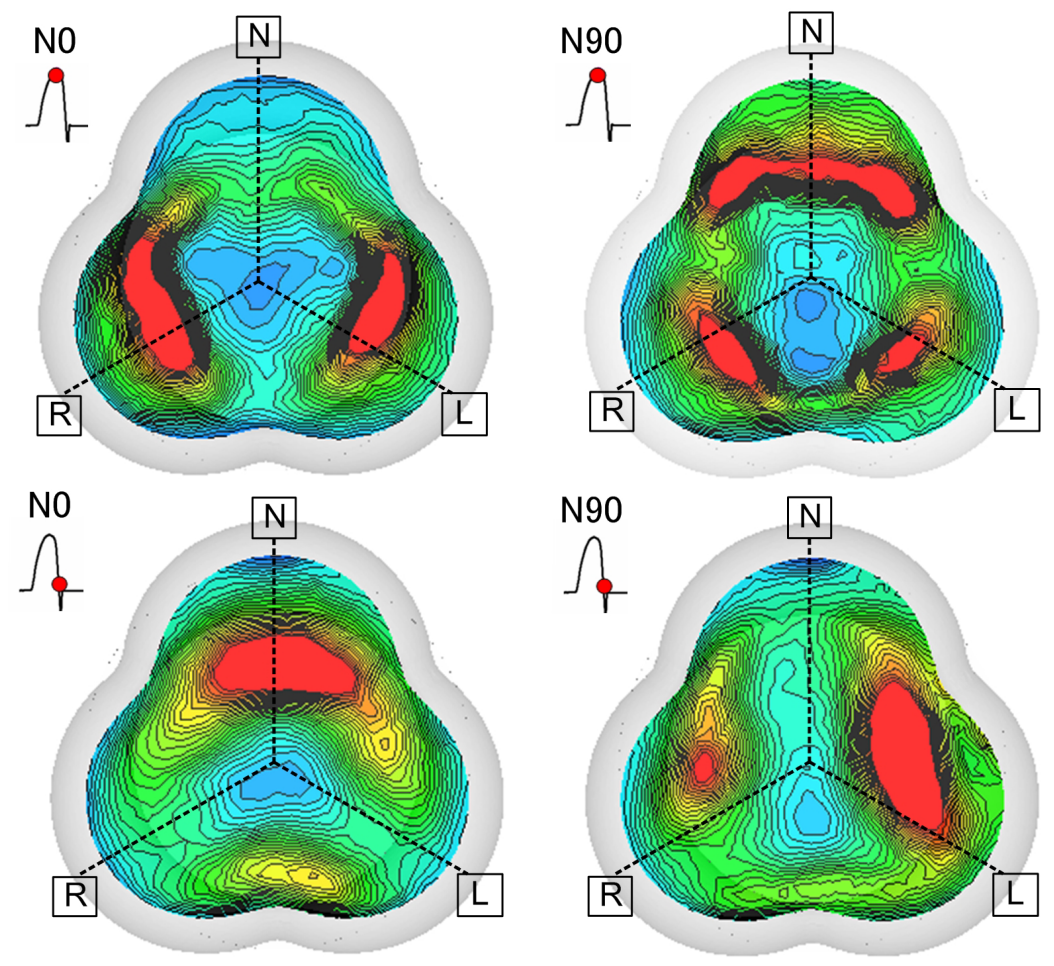

Fig. 11 Comparison of valve orientations (N0 and N90) in turbulent kinetic energy in the vicinity of the sinotubular junction $(z=24 \mathrm{~mm})$. Color coding should be referred to Fig. 10.

\subsection{Turbulent profiles during mid and end-systole}

Figure 10 compares the turbulent kinetic energy of N0 to that of N90 during mid and end-systole. The turbulent kinetic energy was herein adopted because it can represent the associated flow disturbance in a more general meaning. The turbulence was found to vary significantly by altering the valve orientation. During mid-systole, it was localized in the shear layer along with the left and right coronary sinuses (N0), whereas that of $\mathrm{N} 90$ reached the non-coronary sinus. In both cases, the degree of turbulence became more magnified toward the sinotubular junction than in the immediate downstream of valve. Furthermore, the cross-sectional distribution of turbulent kinetic energy revealed the off-axis production of turbulence, indicating the difficulty of determining its principal plane two-dimensionally. During end-systole, the turbulence was localized in the non-coronary sinus (N0), whereas the counterpart reached the left and right coronary sinuses (N90). Figure 11 compares the corresponding value at $z=24 \mathrm{~mm}$ near the sinotubular junction, and the location was assumed to be near the coronary entry of human heart. During a decelerating phase from mid to end-systole, the turbulences in the left and right coronary entries were found to reduce markedly (N0), whereas the counterpart was rather increased with significant asymmetry (N90). In view of the corresponding streamlines (Fig. 9), the left and right sinus vortices were confirmed to become stabilized (N0), whereas the counterparts became further disorganized due to the interference with the asymmetric regurgitant jet (N90).

\section{Discussion}

The present results demonstrated the time-dependent evolutions of sinus flows including mean and turbulent behaviors during a decelerating phase. During mid-systole, each of three sinuses formed a single vortex independently, and the vortices were found to be 
unaffected by the valve orientation (N0 and N90). Toward end-systole, the flow dynamics was revolutionarily changed due to the appearance of regurgitation. The flow feature was found to vary significantly by changing the valve orientation. In the case of N0, the left and right sinus vortices became stabilized toward end-systole, whereas those of N90 became further disorganized. These distinct flow natures were attributed to the hinge of the bileaflet valve. The regurgitation during the decelerating phase was to be directed to the hinge. Although the forward flow was weakened by the adverse pressure gradient, the persistent forward motion was found. Then, the regurgitation was confirmed to coexist with the persistent forward motion by forming a counter-type flow. Counterflow tends to behave unstably due to the hydrodynamic instability of shear layer. This unstable flow pattern resulted in the production of the unexpected turbulence depending on the hinge orientation. If the hinge was aligned in the direction of the non-coronary sinus (N0), no regurgitation disturbed the forward flow. Then, the left and right sinus vortices were dominated by the recirculation from the persistent forward flow. The interaction between the forward and backward fluid momentum was observed only in the non-coronary sinus, but not in the left and right sinuses. On the other hand, if the hinge was aligned in the direction of N-L and N-R commissures (N90), the regurgitation came to be directed to the narrow gap, thereby squeezing the persistent forward flow. This unstable flow configuration resulted in the asymmetric skewed flow pattern with the unexpected turbulence in the left or right coronary sinuses. These results allowed us to better understand the fluid mechanics of aortic sinus. During mid-systole, the presence of aortic sinus forced the forward flow to reverse by reducing the internal pressure. The emerging regurgitation during end-systole was to be directed to the lower pressure region, as if the aortic sinus behaved like a pressure-dependent flow suction and reservoir. The direction of regurgitation could be skewed depending on the orientation of the bileaflet valve (N90). The valve orientation without disturbing the pressure balance formed in each of three sinuses during mid-systole allowed the smooth inflow to the left and right sinuses during end-systole (N0).

Since the onset of the coronary mainstream coincides with the closure of aortic valve, the end-systolic turbulence in the left and right sinuses was speculated to disturb the initiation of coronary stream (N90). The well-organized laminar-like flow was thought to be favorable (N0). Kleine et al. found 1.4 times greater coronary flow rate in the superior orientation. ${ }^{(9)}$ The superior and inferior orientations corresponded to be N0 and N90 in this study, respectively. Therefore, the results presented here advocated the existence of the optimal orientation of the bileaflet valve. Consequently, it was believed that for promoting coronary circulation, sinus vortices should become an organized laminar-like stream toward end-systole. The appearance of turbulence near the coronary entrance should be suppressed. The organized evolution of sinus vortices from mid-systole to end-systole was observed in normal subjects in vivo by MRI velocity mapping. ${ }^{(8)}$ On the other hand, the genesis of cardiovascular diseases (aortic stenosis, valvular insufficiency, etc.) and their surgical treatments including implantable devices (valve-sparing operations, prosthetic valves, valved grafts, etc.) dramatically alter the flow behavior at the aortic sinus of Valsalva. ${ }^{(6-8)}$ Though the clinical implication of these man-made unusual flows remains to be solved, these flow changes were speculated to have the potential of giving an impact on coronary circulation and should be further assessed on it.

The present study successfully characterized the three-dimensional time-dependent evolutions of sinus vortices using a realistic model of the sinus of Valsalva. There are some limitations that should be assessed toward the future. Effects of eccentric inflow, aortic arch, and wall compliance are listed. The skewed helical flow patterns as observed in the ascending aorta toward the arch may be considered. At last, in the present study, three sinuses were recognized to be identically shaped. Although this study treated an ideal condition, the data represented a value of revisiting the complex fluid mechanics of the 
aortic sinus of Valsalva.

\section{Conclusion}

FS-SPIV was developed for performing the three-dimensional analyses of flow dynamics enclosed by complex geometries such as cardiovascular flows. The present study, for the first time, revealed the three-dimensional time-dependent evolutions of sinus vortices during a decelerating phase in vitro. FS-SPIV found that the bileaflet valve orientation affected the evolutional behavior of sinus vortices. The counterflow pattern during end-systole was found to play a key role for determining the stability of left and right sinus vortices. The well-organized laminar-like sinus vortices were only achieved if a pair of valve hinges was aligned in the direction of the non-coronary sinus, which were believed to promote the coronary circulation. The results presented here advocated the existence of the optimal orientation of the bileaflet valve. This study demonstrated the first experimental results of three-dimensional validated velocity data sets of aortic flows by considering the realistic valve opening and closing dynamics. These data are also useful to validate and optimize the numerical code of computational fluid dynamics.

\section{Acknowledgements}

This research was supported by the Biomedical Engineering Project Research at Waseda Research Institute for Science and Engineering, Waseda University, Tokyo. Experiments were conducted in collaboration with the Laser Flow Diagnostic Laboratory at The Commonwealth Scientific and Industrial Research Organization (CSIRO), Australia. The authors with to express their sincere thanks for CSIRO.

\section{References}

(1) Bellhouse, B. J. and Talbot, L., The fluid mechanics of the aortic valve. Journal of Fluid Mechanics, Vol.35, No.4, pp.721-735, 1969.

(2) Brandt, J., Jögi, P., and Lührs, C., Sinus of Valsalva aneurysm obstructing coronary arterial flow: case report and collective review of the literature. European Heart Journal, Vol.6, pp.1069-1073, 1985.

(3) Paulis, R. D., Tomai, F., Bertoldo, F., Ghini, A. S., Scaffa, R., Nardi, P., and Chiariello, L., Coronary flow characteristics after a Bentall procedure with or without sinuses of Valsalva. European Journal of Cardio-thoracic Surgery, Vol.26, pp.66-72, 2004.

(4) Demers, P. and Miller, D. C., Simple modification of "T. David-V" valve-sparing aortic root replacement to create graft pseudosinuses. Annals of Thoracic Surgery, Vol.78, No.4, pp.1479-1481, 2004.

(5) Van't Veer, M., Van Straten, B., Van de Vosse, F., and Pijls, N., Influence of orientation of bi-leaflet valve prostheses on coronary perfusion pressure in humans. Interactive Cardiovascular and Thoracic Surgery, Vol.6, pp.588-592, 2007.

(6) Botnar, R., Nagel, E., Scheidegger, M. B., Pedersen, E. M., Hess, O., and Boesiger, P., Assessment of prosthetic aortic valve performance by magnetic resonance velocity mapping. Magnetic Resonance Materials in Physics, Biology and Medicine, Vol.10, pp.18-26, 2000.

(7) Markl, M., Draney, M. T., Miller, D. C., Levin, J. M., Williamson, E. E., Pelc, N. J., Liang, D. H., and Herfkens, R. J., Time-resolved three-dimensional magnetic resonance velocity mapping of aortic flow in healthy volunteers and patients after valve-sparing aortic root replacement. The Journal of Thoracic and Cardiovascular Surgery, Vol.130, pp.456-463, 2005. 
(8) Kvitting, J-P. E., Ebbers, T., Wigstöm, L., Engvall, J., Olin, C. L., and Bolger, A. F., Flow patterns in the aortic root and the aorta studied with time-resolved, 3-dimensional, phase-contrast magnetic resonance imaging: implications for aortic valve-sparing surgery. The Journal of Thoracic and Cardiovascular Surgery, Vol.127, pp.1602-1607, 2004.

(9) Kleine, P., Scherer, M., Abdel-Rahman, U., Klesius, A. A., Ackermann, H., and Moritz, A., Effect of mechanical aortic valve orientation on coronary artery flow: comparison of tilting disc versus bileaflet prostheses in pigs. The Journal of Thoracic and Cardiovascular Surgery, Vol.124, No.5, pp.925-932, 2002.

(10) Laas, J., Kleine, P., Hasenkam, M. J., and Nygaard, H., Orientation of tilting disc and bileaflet aortic valve substitutes for optimal hemodynamics. The Annals of Thoracic Surgery, Vol.68, pp.1096-1099, 1999.

(11) Klein, P., Perthel, M., Hasenkam, J. M., Nygaard, H., Hansen, S. B., and Laas, J., Downstream turbulence and high intensity transient signals (HITS) following aortic valve replacement with Medtronic Hall or St. Jude Medical valve substitutes. European Journal of Cardio-thoracic Surgery, Vol.17, pp.20-24, 2000.

(12) Yoganathan, A. P., Chaux, A., Gray, R. J., Woo, Y., DeRobertis, M., Williams, F. P., and Matloff, J. M., Bileaflet, tilting disc and porcine aortic valve substitutes: in vitro hydrodynamic characteristics. Journal of the American College of Cardiology, Vol.3, No.2, pp.313-320, 1984.

(13) Hasenkam, J. M., Giersiepen, M., and Reul, H., Three-dimensional visualization of velocity fields downstream of six mechanical aortic valves in a pulsatile flow model. Journal of Biomechanics, Vol.21, No.8, pp.647-661, 1988.

(14) Browne, P., Ramuzat, A., Saxena, R., and Yoganathan, A. P., Experimental investigation of the steady flow downstream of the St. Jude bileaflet heart valve: a comparison between Laser Doppler Velocimetry and Particle Image Velocimetry Techniques. Annals of Biomedical Engineering, Vol.28, pp.39-47, 2000.

(15) Akutsu, T. and Fukuda, T., Time-resolved particle image Velocimetry and laser Doppler anemometry study of the turbulent flow field of bileaflet mechanical mitral prostheses. Journal of Artificial Organs, Vol.8, pp.171-183, 2005.

(16) Dyverfeldt, P., Kvitting, J-P. E., Sigfridsson, A., Engvall, J., Bolger, A. F., and Ebbers, T., Assessment of fluctuating velocities in disturbed cardiovascular blood flow: in vivo feasibility of generalized phased-contrast MRI. Journal of Magnetic Resonance Imaging, Vol.28, pp.655-663, 2008.

(17) Schmidtke, C., Matthias, J. F., Hueppe, M., Noetzold, A., and Sievers, H-H., Size and distensibility of the aortic root and aortic valve function after different techniques of the Ross procedure. The Journal of Thoracic and Cardiovascular Surgery, Vol.119, pp.990-997, 2000.

(18) Reul, H., Vahlbruch, A., Giersiepen, M., Schmitz-Rode, T. H., Hirtz, V., and Effert, S., The geometry of the aortic root in health, at valve disease and after valve replacement. Journal of Biomechanics, Vol.23, No.2, pp.181-191, 1990.

(19) Nichols, W. W. and O'Rourke, M. F., McDonald's blood flow in arteries. London: Hodder Arnold, 1998.

(20) Johnson, K., Sharma, P., and Oshinski, J., Coronary artery flow measurement using navigator echo gated phase contrast magnetic resonance velocity mapping at 3.0 tesla. Journal of Biomechanics, Vol.41, No.3, pp.595-602, 2008.

(21) Chien, S., Shear dependence of effective cell volume as a determinant of blood viscosity. Science, Vol.168, No.3934, pp.977-979, 1970.

(22) Marassi, M., Castellini, P., Pinotti, M., and Scalise, L., Cardiac valve prosthesis flow performances measured by 2D and 3D-Stereo Particle Image Velocimetry. Experiments in Fluids, Vol.36, pp.176-186, 2004.

(23) Kaminsky, R., Kallweit, S., Weber, H-J., Claessens, T., Jozwik, K., and Verdonck, P., Flow 
visualization through two types of aortic prosthetic heart valves using Stereoscopic High-speed Particle Image Velocimetry. Artificial Organs, Vol.31, No.12, pp.869-879, 2007.

(24) Leo, H. L., Dasi, L. P., Carberry, J., Simon, H. A., and Yoganathan, A. P., Fluid dynamic assessment of three polymeric heart valves using particle image Velocimetry. Annals of Biomedical Engineering, Vol.34, No.6, pp.936-952, 2006.

(25) Soloff, S. M., Adrian, R. J., and Liu, Z. C., Distortion compensation for generalized stereoscopic particle image velocimetry. Measurement Science and Technology, Vol.8, pp.1441-1454, 1997.

(26) Raffel, M., Willert, C., and Kompenhans, J., Particle Image Velocimetry: A Practical Guide. Springer, 1998.

(27) Yoon, J-H. and Lee, S-J., Direct comparison of 2D PIV and stereoscopic PIV measurements. Measurement Science and Technology, Vol.13, pp.1631-1642, 2002.

(28) Westerweel, J., Theoretical analysis of the measurement precision in particle image velocimetry. Experiments in Fluids, pp.S3-S12, 2000. 